\title{
Effect of carnosine supplementation on serum adipokines in overweight and obese adults: a pilot randomised trial
}

Estifanos Baye, Jozef Ukropec, Maximilian PJ de Courten, Giancarlo Aldini, Kirsty Wilson, Magda Plebanski, Barbara Ukropcova, Barbora de Courten

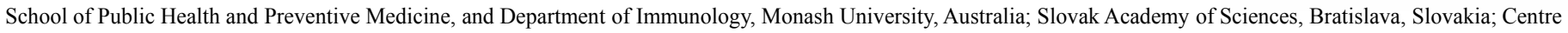
for Chronic Disease, Victoria University, Australia; Department of Pharmaceutical Sciences, Università degli Studi di Milano, Italy

Background

- Adipokines play a role in the pathophysiology of insulin resistance, type 2 diabetes $(\mathrm{T} 2 \mathrm{DM})$ and cardiovascular diseases (CVD).

- We have previously shown that carnosine, an over-the-counter food supplement, prevented worsening of glucose metabolism in overweight and obese individuals.

- The effect of carnosine on serum adipokines has thus far not been investigated.

\section{Hypothesis}

We hypothesised that carnosine supplementation improves serum adipokines in overweight and obese otherwise healthy adults, compared to placebo.

\section{Aim}

To determine the effect of carnosine supplementation on serum adipokine levels in non-diabetic overweight and obese individuals.

\section{Methods}

- Study design: Randomised, double-blind, placebo controlled trial

- Sample size: 30 overweight and obese individuals (BMI $\geq 25 \mathrm{~kg} / \mathrm{m}^{2}$ )

- Eligibility criteria: Non-diabetic, no sign of acute or chronic infection, not taking any medication or food supplements, nonsmokers, healthy according to a physical examination and routine blood analyses

- Intervention: $2 \mathrm{~g}$ carnosine daily (administered orally divided in two equivalent doses) for 12 weeks

- Comparator: Matching placebo

- Measurements:

$-75 \mathrm{~g}$ oral glucose tolerance test performed

Serum lipid levels determined using commercial diagnostic kits (Roche, Germany)

Serum adipokines were quantified by the LEGENDplex ${ }^{\text {TM }}$ Human Metabolic 1 bead-based multiplex assay (BioLegend).

Triple quadrupole mass spectrometer (TSQ QUANTUM ULTRA, Thermo Scientific, Italy) used to measure urinary carnosine levels.

\section{$\underline{\text { Results }}$}

Participant Baseline Characteristics

- 8 participants excluded ( 3 protocol violation \& 5 high markers of inflammation).

- 22 participants (13 in carnosine group, 9 in placebo group) included in the analysis.

- Overall mean age: $43.4 \pm 8.1$ years; BMI: $31.3 \pm$ $4.4 \mathrm{~kg} / \mathrm{m}^{2}$; waist-to-hip ratio: $0.89 \pm 0.06$

- Groups did not differ in baseline cardiometabolic and carnosine measures.

- There were no differences in serum adipokines between groups at baseline.

\section{Effect of carnosine on serum adipokines}

- Carnosine supplementation decreased resistin levels compared to placebo (mean change difference: $-56.15 \pm 29.08 \mathrm{ng} / \mathrm{ml}, \mathrm{p}=0.04$ ) (Fig 1).

- A trend for reduction in leptin levels was observed after carnosine supplementation compared to placebo (mean change difference: $-111.74 \pm 57.66 \mathrm{ng} / \mathrm{ml}, \mathrm{p}=0.06$ ) (Fig 2).

- Adiponectin and adipsin levels were not different between carnosine and placebo groups (all $\mathrm{p}>0.1$ )

Fig 1. Effect of carnosine on serum resistin levels

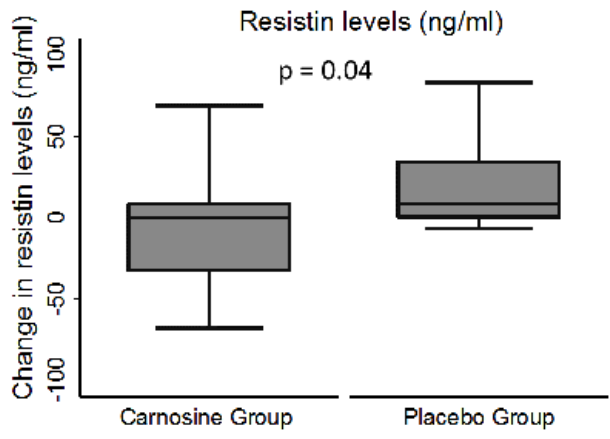

Fig 2. Effect of carnosine on serum leptin levels

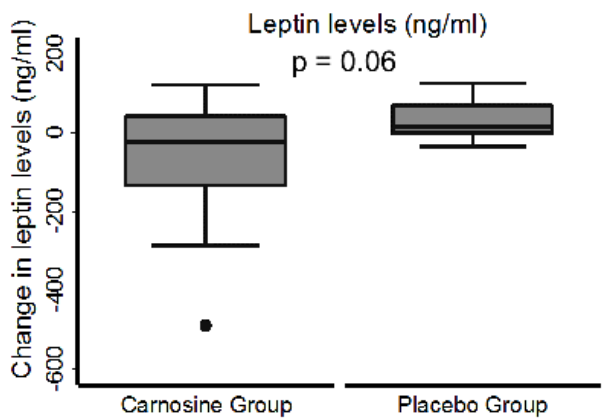

$\underline{\text { Relationship between serum adipokines \& }}$ urinary carnosine levels

- Change in resistin and leptin levels were inversely correlated with change in:

- Urinary carnosine $(\mathrm{r}=-0.75, \mathrm{p}=0.001 ; \mathrm{r}=$ $-0.71, \mathrm{p}=0.0001$, respectively) (Fig $3 \& 4$ )

Carnosine-propanal $(\mathrm{r}=-0.56, \mathrm{p}=0.005$; $\mathrm{r}=-0.63, \mathrm{p}=0.002$, respectively)

Carnosine-propanol ( $r=-0.61, p=0.002$; $r=-0.61, p=0.002$, respectively)

Fig 3. Relationship between resistin and urinary carnosine levels

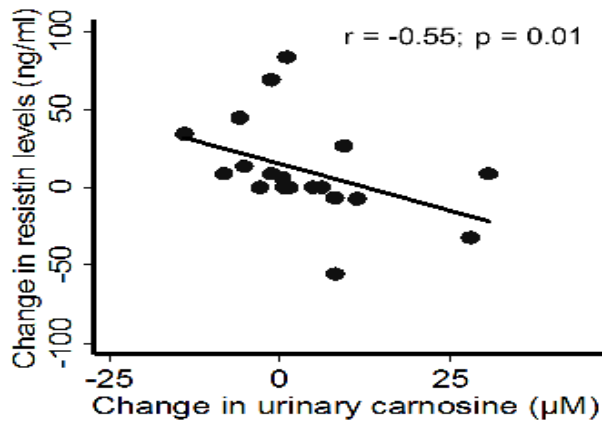

Fig 4. Relationship between leptin and urinary carnosine levels

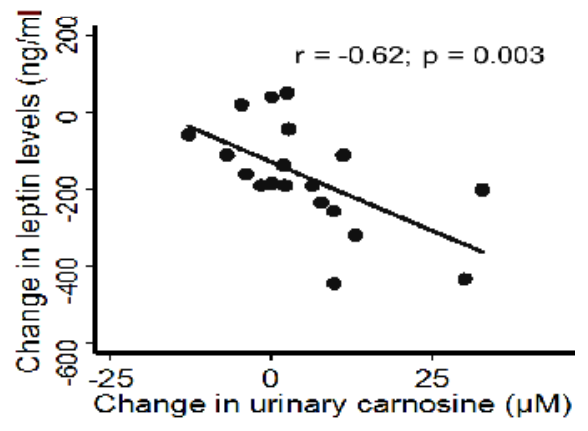

- The observed correlations were remained significant after adjusting for age, sex and BMI (all p < 0.05) except for resistin and carnosine-propanol levels ( $\mathrm{p}=0.1$ ).

- Change in resistin or leptin levels were not associated with any cardiometabolic parameters (data not shown).

\section{Conclusions}

- Carnosine supplementation reduced serum resistin and leptin levels in overweight and obese otherwise healthy adults.

\section{$\underline{\text { References }}$}

- Ryo M, et al, Circ J., 2004

- de Courten B, et al, Obesity, 2016

- Aldini G, et al, J Cell Mol Med, 2011

- Lee YT, et al, Eur J Pharmacol, 2005 